2. Short-termism. Interventions are designed in crisis or as simple 'quick wins', to the detriment of long-term, sustainable approaches that address coherent sets of issues facing populations or health systems

3. Neglect of underlying social, structural and commercial determinants of the health of populations The SDGs shift the focus towards tackling inter-dependent and often intractable problems that are both local and global, and demand a change in the approaches to global health and SRH interventions. Simply placing Universal Health Coverage as the center-piece of the SDGs is inadequate. Instead, emphasis is needed on greater participation by those most affected, and promoting coherence and integration beyond SRH programs or the health sector. Practical approaches would address linkages with economic opportunity, gender discrimination, education, water $\&$ sanitation, food security, environment and resource management, public safety, conflict management, disaster preparedness and other areas of development.

Disclosure No significant relationships.

\section{PL12 - OVERVIEW ON THE DEVELOPMENT OF POINT-OF-CARE TESTS FOR DETECTION OF ANTIMICROBIAL RESISTANCE - CHALLENGES AND OPPORTUNITIES}

\author{
Wednesday, July 17, 2019 \\ 8:15 AM - 8:50 AM
}

\section{PL12.1 OVERVIEW ON THE DEVELOPMENT OF POINT-OF-CARE TESTS FOR DETECTION OF ANTIMICROBIAL RESISTANCE - CHALLENGES AND OPPORTUNITIES}

Johan Melendez*. Johns Hopkins University, Medicine - Infectious Diseases, Baltimore, USA

10.1136/sextrans-2019-sti.15

The increasing rates of gonorrhea infections worldwide and the evolving profile of antimicrobial resistance has prompted global public health concerns regarding the successful management of gonorrhea. In the absence of new antimicrobials, the use of point-of-care (POC) tests for identifying infected individuals and guiding precision treatment has been proposed as a strategy to mitigate the effects of antimicrobial resistant (AMR) gonorrhea. However, despite advancements in nucleic acid amplification technologies, there is no commercially available test for antimicrobial susceptibility testing (AST) that we can be implemented at the POC. Genotyping assays for prediction of antimicrobial susceptibility have been developed and are beginning to be implemented in the clinical setting for precision treatment. However, these assays are not at the POC and cannot reliably be used to predict susceptibility to all antimicrobials. While still in the early stages of development, novel phenotypic approaches targeting antimicrobial-reactive markers have shown promise as an approach on which to build rapid AST assays. Due to their speed, diagnostic performance, and portability, microfluidic-based technologies meet several of the diagnostic criteria for the development of rapid
AST assays which could be implemented at the POC. Implementation in the clinical setting of these AST POC tests is another major challenge which should be evaluated and implementation strategies developed concurrently to test development.

Disclosure No significant relationships.

\section{PL13 - THE ROLE OF KEY POPULATION-LED HEALTH SERVICES IN ADDRESSING THE CHALLENGE OF STI AND HIV}

\section{Wednesday, July 17, 2019 8:50 AM - 9:25 AM}

\section{PL13.1 THE ROLE OF KEY POPULATION-LED HEALTH SERVICES IN ADDRESSING THE CHALLENGE OF STI AND HIV}

Rena Janamnuaysook* . The Thai Red Cross AIDS Research Center, Bangkok, Thailand

10.1136/sextrans-2019-sti.16

Community engagement are essential in HIV responses. Key population (KP) play important roles on task sharing as one of methods of strengthening and expanding health workforce to rapidly increase access to HIV and STI services. Certain tasks are shared, where appropriate, from qualified health professionals to trained KP lay providers with certified trainings and particular practices in order to make more efficient use of human resources for health among members of KP.

Key populations in Thailand - men have sex with men (MSM), transgender women (TGW) and sex workers - established community health clinics as a key population-led health service (KPLHS) model to provide HIV and STI services, including peer outreach, HIV counseling, finger prick blood and specimen sample collections for HIV testing and STI screening, and dispensing of pre-exposure prophylaxis (PrEP) and post-exposure prophylaxis (PEP) as well as linkage to care and treatment.

It was demonstrated that KPLHS successfully increased access to STI and HIV prevention, care and treatment services among KP. Tangerine Community Health Center, the first transgender-led clinic in Bangkok, provides comprehensive health services and gender-affirming hormone treatment to transgender women with high HIV and syphilis prevalence. In addition, SWING Foundation, led by sex worker communities, caters HIV and STI services to male sex workers located in Bangkok and Pattaya. Trained KP lay providers from Rainbow Sky Association of Thailand (RSAT) offers HIV testing, syphilis screening, PrEP and PEP to young MSM and those who engage in sex work. The impact of KPLHS contributed to $42 \%$ of HIV testing and 52\% of PrEP uptake among KP nationally.

KPLHS as a promising model to accelerate an end to the AIDS epidemic must be expanded. Laws and regulations should be amended to allow implementation at scale. The government should be committed to community health financing to sustain KPLHS.

Disclosure No significant relationships. 